\title{
Key miRNAs associated with memory and learning disorder upon exposure to sevoflurane determined by RNA sequencing
}

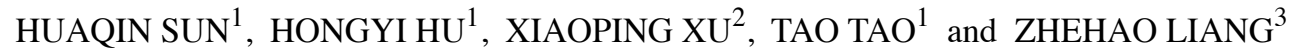 \\ ${ }^{1}$ Department of Anesthesiology, The First Affiliated Hospital of Zhejiang Chinese Medical University, \\ Hangzhou, Zhejiang 310006; ${ }^{2}$ Laboratory Animal Research Center/Institute of Comparative Medicine, Zhejiang Chinese \\ Medical University, Hangzhou, Zhejiang 310053; ${ }^{3}$ Department of Ultrasound, The First Affiliated Hospital of \\ Zhejiang Chinese Medical University, Hangzhou, Zhejiang 310006, P.R. China
}

Received October 28, 2019; Accepted April 15, 2020

DOI: $10.3892 / \mathrm{mmr} .2020 .11199$

\begin{abstract}
The study aimed to identify differentially expressed microRNAs (miRNAs/miRs) and explore the mechanisms governing impaired memory and learning ability in developing brains exposed to sevoflurane. A total of six 7-day-old male ICR mice were randomly assigned into the sevoflurane anesthesia group (treated with $2.4 \%$ sevoflurane) or control group (treated with normal saline solution at the same dose). After 14 days, the mice were subjected to a Morris water maze experiment. Then, the animals were sacrificed and hippocampus tissues were isolated. RNAs in hippocampus tissues were sequenced and the differential miRNA expression profiles were identified by a bioinformatics approach. The learning and memory function of mice were significantly affected by sevoflurane exposure. A total of 18 miRNAs were found to be significantly affected by sevoflurane administration. Their target genes clustered into different functional groups, such as 'dephosphorylation', 'vesicle localization' and the 'Wnt signaling pathway'. miR-101b-3p was closely related with 'chromatin binding' and 'protein serine/threonine kinase activity'. The most represented pathways for miRNAs included 'neuroactive ligand-receptor interaction' (miR-1187), 'long-term depression' (miR-425-5p), 'FoxO signaling pathway' (miR-425-5p) and the 'neurotrophin signaling pathway' (miR-467a-3p). miR-467a-3p (degree=89), miR-101b-3p (degree=59), and miR-1187 (degree=51) were the hub nodes in the miRNA regulatory network. The Wnt signaling pathway, miR-467a-3p, miR-1187 and miR-101b-3p may be therapeutic targets for preventing cognitive impairments induced by sevoflurane.
\end{abstract}

Correspondence to: Dr Zhehao Liang, Department of Ultrasound, The First Affiliated Hospital of Zhejiang Chinese Medical University, 54 Youdian Road, Shangcheng, Hangzhou, Zhejiang 310006, P.R. China

E-mail:drugabc@163.com

Key words: sevoflurane, RNA sequencing, differentially expressed microRNA, function enrichment analysis, pathway

\section{Introduction}

Sevoflurane, a volatile anesthetic agent, is widely used for pediatric anesthesia in the clinic, and is characterized by rapid onset and offset, and low airway irritation and blood/gas partition coefficient (1). Previous evidence has suggested that long-term exposure to volatile anesthetics have side effects on brain development. It is reported that sevoflurane administration can impair memory processes, spatial memory, and the ability of the hippocampus to learn tasks in human and animal brains (2-4). Thus, concerns regarding the side effects of sevoflurane in children undergoing surgery have been highlighted.

An increasing number of studies have explored the molecular mechanisms that underlie the effects of sevoflurane exposure on the brain (1,4-6). The neurotoxicity of sevoflurane was shown to decrease guanylate kinase concentration in glutamatergic synapses in the development of rat brains (1). Sevoflurane-induced memory impairment is closely related with the suppression of glycogen synthase in the hippocampus (4). In addition, memory impairment following sevoflurane exposure is also reported to occur due to the decreased cytosolic calcium concentration and $\mu$-calpain activity (5). Previous studies suggest that sevoflurane exposure alters the expression of genes involved in cognitive function-related metabolic pathways (6). Another previous study suggested that sevoflurane exposure leads to changes in the expression of receptors and enzymes involved in amyloid $\beta$ clearance, which contributes to Alzheimer's disease development $(7,8)$. The cognitive dysfunction induced by sevoflurane has been shown to be relieved by mediating the Toll-like receptor 4/myeloid differentiation primary response $88 / \mathrm{NF}-\kappa \mathrm{B}$ signaling pathway (8).

MicroRNAs (miRNAs/miRs) have been reported to be involved in neuropsychiatric disorders and impairments in cognitive function $(9,10)$. However, the role of miRNAs in sevoflurane-induced neurotoxicity has not been fully clarified. The binding of Wnt ligands to receptors/co-receptors promotes Wnt signaling activation (11). Previous studies have revealed that the WNT signaling pathway is one of the main signaling pathways involved in numerous types of disease, including osteoporosis, cancer and diabetes $(12,13)$. Moreover, it has been identified that miRNAs serve important regulatory roles 
in the Wnt signaling pathway (14). However, the association between miRNAs and Wnt signaling in sevoflurane-induced neurotoxicity remains largely unknown. In this paper, miRNA expression in hippocampus samples from newborn mice was characterized by microarray technology. The miRNAs with differential expression were determined, followed by function and pathway enrichment analysis. The aim of this study was to explore the mechanism underlying the effect of sevoflurane on the developing brain and facilitates the discovery of safe anesthetic strategies.

\section{Materials and methods}

Animals. A total of four 15-day-old pregnant Institute of Cancer Research (ICR) mice were purchased from Shanghai Sipubikai Laboratory Animal Co., Ltd. The pregnant mice were maintained in an animal house at a temperature of $20 \pm 2^{\circ} \mathrm{C}$ and humidity of $55 \pm 5 \%$, with a 12 -h light/dark cycle, and free access to food and water. After parturition, the healthy 7-day-old ICR mice were used for further analysis (15).

Approval was obtained from Animal Care and Ethics Committee of Zhejiang Chinese Medical University and all the animal procedures were performed according to the ethical standards.

Experimental groups. A total of six 7-day-old male ICR mice (weight, 250 $\pm 10 \mathrm{~g}$ ) were randomly assigned into two groups ( $n=3 /$ group): The Sevoflurane group and Control group. Mice in the Sevoflurane group were administered with $2.4 \%$ sevoflurane for $6 \mathrm{~h}$ consecutively between 9:00 am and 3:00 pm according to previously described methods (16). Animals in the Control group were treated with the same dose of normal saline solution via a venous catheter at a rate of $1.0 \mathrm{ml} / \mathrm{h}$. After treatment, all the animals were breast-fed for 14 days followed by a Morris water maze test (17).

Morris water maze. The water maze was comprised of a cylindrical pool (height, $50 \mathrm{~cm}$; diameter, $80 \mathrm{~cm}$ ) and a platform (diameter, $10 \mathrm{~cm}$ ). The water surface was $2 \mathrm{~cm}$ higher than the platform and water was maintained at $22 \pm 0.5^{\circ} \mathrm{C}$. The animals were trained to find the platform and stayed for $2 \mathrm{~min}$ in the water maze twice/day for two consecutive days. The pool was divided into four quadrants. Rats were randomly delegated into the four quadrants, and then were placed in the water, facing the wall of the pool. If the mice failed to find the platform within $2 \mathrm{~min}$, mice were placed on the platform for $20 \mathrm{sec}$. The second trial was conducted after a delay of 5-10 sec. The Morris water maze experiments were monitored by videos recorded on a computer. After training, the time for mice to reach to platform (escape latency) was recorded within $120 \mathrm{sec}$. Then, a probe trial was conducted after removing platform from the pool, and the mice were placed in a given quadrant and allowed to explore the maze for $120 \mathrm{sec}$. The cross-platform path length, percentage of the total trial path length that passed through the platform location, duration that the mouse stayed on the platform and the number of times across the platform were recorded.

RNA isolation. After the multiple behavioral tests on each mouse, mice were sacrificed, and hippocampal tissues were isolated. Total RNA was extracted by TRIzol ${ }^{\circledR}$ reagent (Invitrogen; Thermo Fisher Scientific, Inc.) according to the manufacturer's instructions. The purity of RNA was detected by a NanoDrop ND-2000 (NanoDrop Technologies; Thermo Fisher Scientific, Inc.) and the quality was assessed by an Agilent Bioanalyzer 2100 (Agilent Technologies, Inc.).

miRNA profiling with microarray. Total RNA samples with an RNA integrity number $>9$ were used for microarray analysis. RNA (250 ng) from each sample was dephosphorylated, degenerated and labeled with Cyanine-3-CTP (Cy3) according to the manufacturer's instructions for the Human miRNA Microarray kit (Agilent Technologies, Inc.). After purifying, RNA was hybridized to gene arrays at $55^{\circ} \mathrm{C}$ for $20 \mathrm{~h}$ and scanned on an Agilent Scanner G2505C system (Agilent Technologies, Inc). The raw data were exported to .txt format by Agilent Feature Extraction (FE) software (version 9.5.3; Agilent Technologies, Inc.) for further analysis.

Datapreprocessinganddifferentialexpressionanalysis. The text format data were transformed by Affy package v1.50.0 (18) in R (http://www.bioconductor.org/packages/release/bioc/html/affy. html) and preprocessed by the robust multi-array average method $(19,20)$, including background correction, normalization and expression calculation.

Comparedwithcontrols, themiRNAswithdifferentialexpression in the Sevoflurane group were assessed by limma v3.26.9 (http://bioconductor.org/packages/release/bioc/html/limma. html) (21). The P-values and fold change (FC) in expression of miRNAs were calculated. $\mathrm{P}<0.01$ and $\mid \log _{2} \mathrm{FCl}>0.263$ was considered to indicate a statistically significant difference $(22,23)$. Hierarchical clustering of differentially expressed miRNAs was performed by pheatmap package version 1.0.8 (https://cran.r-project.org/web/packages/pheatmap).

Prediction of miRNA target genes. The differentially expressed miRNAs were uploaded to the miRNA-Gene Targets module of miRWalk 2.0 (http://zmf.umm.uni-heidelberg.de/apps/zmf/mirwalk2/miR retsys-self.html) (24,25). Subsequently, the miRNA targets were also predicted by miRanda version 3.0 (http://cbio.mskcc.org/microrna), miRDB version 4.0 (http://mirdb.org/miRDB), PITA (http://genie.weizmann.ac.il/pubs/mir07/mir07_data.html), RNA22 version 2.0 (https://cm.jefferson.edu/rna22), RNAhybrid version 2.12 (https://bibiserv.cebitec.uni-bielefeld.de/rnahybrid) and TargetScan version 7.0 (http://www.targetscan.org/) databases.

In order to explore the biological functions of differentially expressed miRNAs, the predicted target genes were subjected to Gene Ontology (GO) (26) and Kyoto Encyclopedia of Genes and Genomes (KEGG) pathway enrichment analysis (27) using clusterProfiler version 2.4.3 (https://bioconductor. org/packages/release/bioc/html/clusterProfiler.html) (28). $\mathrm{P}<0.05$ was set as the threshold value.

miRNA-mRNA regulatorynetwork construction. The predicted miRNA-target pairs were identified, and the miRNA-target regulatory network was constructed by Cytoscape version 2.8 software (29). The topology of the miRNA regulatory network was analyzed and the hub nodes with significant degrees were screened out. 
Table I. Sevoflurane exposure affects the learning and memory ability in mice.

\begin{tabular}{llcrc}
\hline Phases of trial & \multicolumn{1}{c}{ Index } & Sevoflurane group & Control group & P-value \\
\hline Learning trial & Mean escape latency, sec & $119.33 \pm 4.04$ & $13.67 \pm 2.08$ & $<0.001$ \\
Probe trial & Cross-platform path length, mm & $71.19 \pm 2.45$ & $92.06 \pm 2.09$ & $<0.001$ \\
& Percentage of total path length in platform & $0.62 \pm 0.02$ & $0.83 \pm 0.03$ & $<0.001$ \\
& Platform duration, sec & $0.65 \pm 0.04$ & $0.83 \pm 0.06$ & 0.0028 \\
& Platform entries, number of events & $1.33 \pm 0.58$ & $2.67 \pm 0.58$ & 0.47 \\
\hline
\end{tabular}

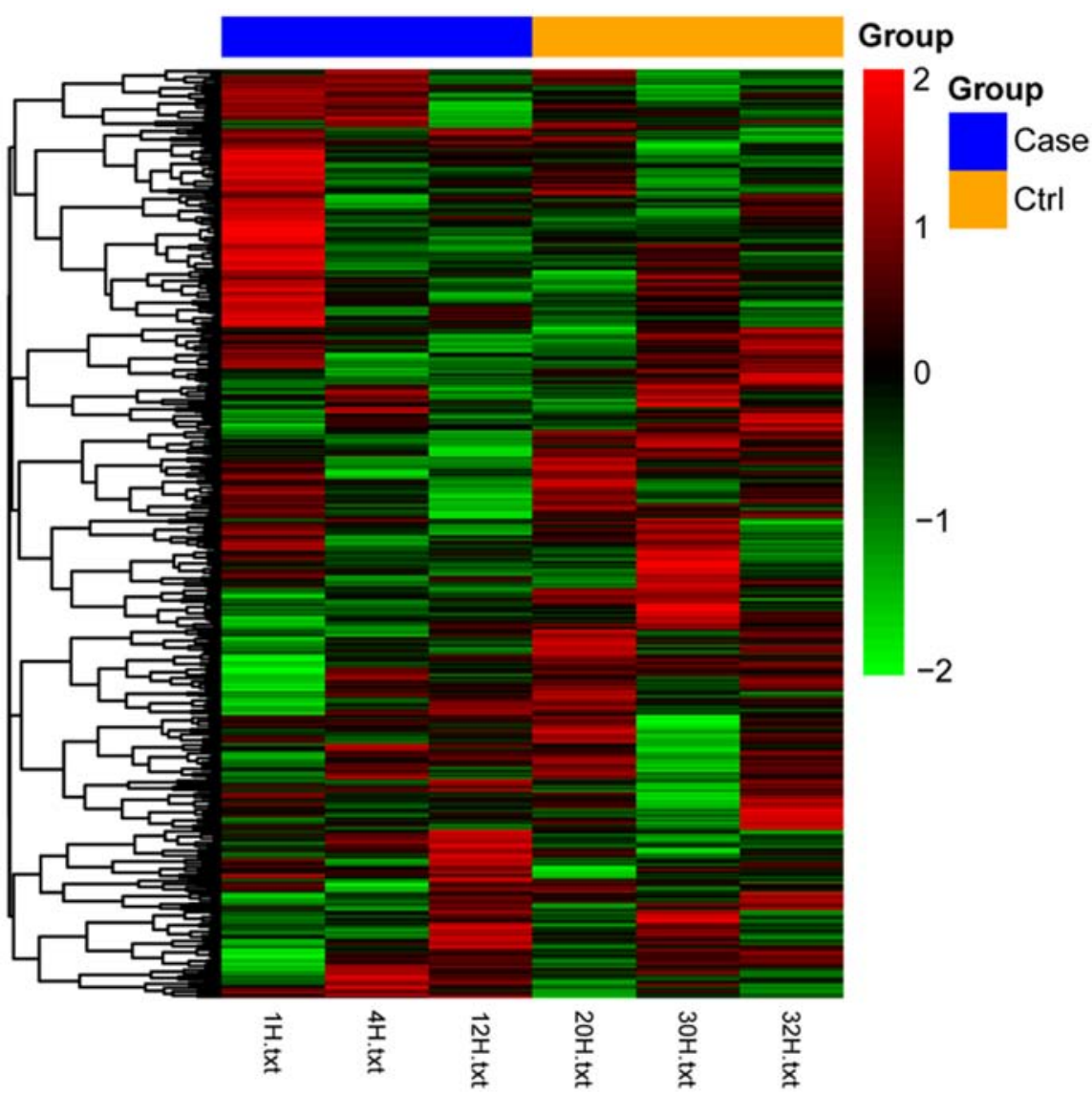

Figure 1. Heatmap of differentially expressed microRNAs in the sevoflurane-exposed group relative to controls. Each column represents samples and each row represents miRNAs. Red represents upregulated miRNAs and green represents downregulated miRNAs. The first four columns represent the level of expression in the sevoflurane group, while the last four columns represent the level of corresponding miRNA expression in the control group.

Statistical analysis. The data are expressed as mean $\pm \mathrm{SD}$. Differences between groups were compared using t-tests. $\mathrm{P}<0.05$ was considered to indicate a statistically significant difference.

\section{Results}

Sevoflurane exposure affects learning and memory function in newborn mice. During learning, following exposure to sevoflurane, mice in the Sevoflurane group $(119.33 \pm 4.04 \mathrm{sec})$ showed longer mean latency time to reach the platform than those in the Control group (13.67 \pm 2.08 sec; $\mathrm{P}<0.001$; Table I). During the probe trial, the cross-platform path length was significantly shorter in sevoflurane-treated mice $(71.19 \pm 2.45 \mathrm{~mm})$, compared with Controls $(92.06 \pm 2.09 \mathrm{~mm} ; \mathrm{P}<0.001)$. Similarly, the percentage of the total path length that went through the platform location was significantly lower in sevoflurane-exposed mice $(0.62 \pm 0.02)$ compared with the Control group $(0.83 \pm 0.03$; $\mathrm{P}<0.001)$. The platform duration in mice exposed to sevoflurane was significantly declined $(0.65 \pm 0.04 \mathrm{sec})$ compared with the Control group $(0.83 \pm 0.06 \mathrm{sec} ; \mathrm{P}=0.0028)$. In addition, the mean number of times across platform for mice treated with sevoflurane $(1.33 \pm 0.58)$ was not significantly lower than the Control group $(2.67 \pm 0.58 ; \mathrm{P}=0.47)$.

Data preprocessing. Based on the raw data, expression information for 52,044 miRNAs was available. The expression profiles of 49,880 miRNAs were obtained, followed by data preprocessing. After miRNA overlaps were removed, the mean expression values of 1,247 mature miRNAs were calculated following previously published descriptions (30). 

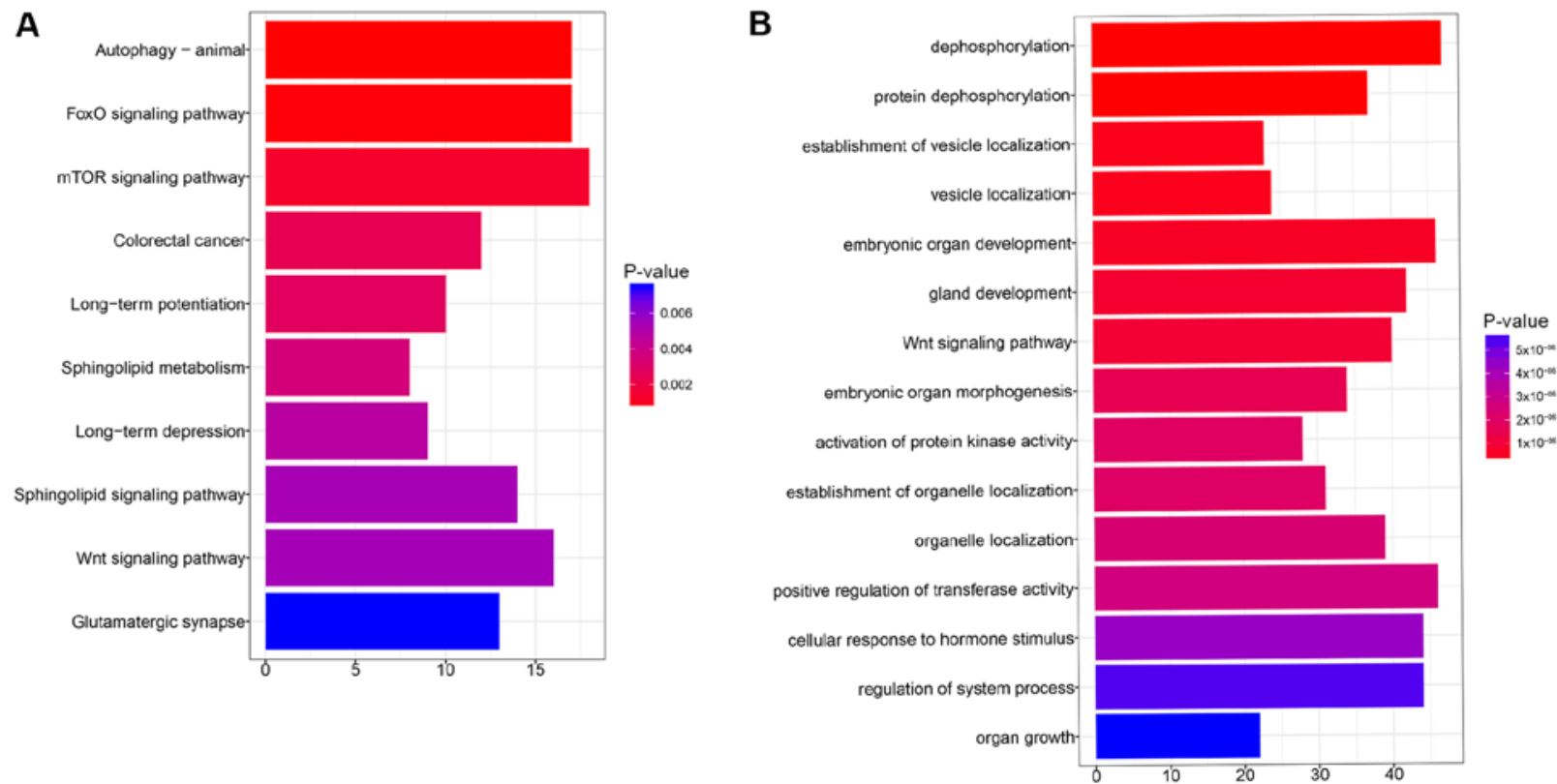

Figure 2. Enrichment analysis of targets associated with sevoflurane. (A) KEGG pathways significantly enriched by gene targets of differentially expressed miRNAs. y-axis, statistically enriched pathway; x-axis, the number of differentially expressed genes in each pathway. (B) GO functions significantly enriched by gene targets of differentially expressed microRNAs. $y$-axis, statistically enriched GO biological process terms; $\mathrm{x}$-axis, the number of differentially expressed genes in each GO term. KEGG, Kyoto Encyclopedia of Genes and Genomes; GO, Gene Ontology.

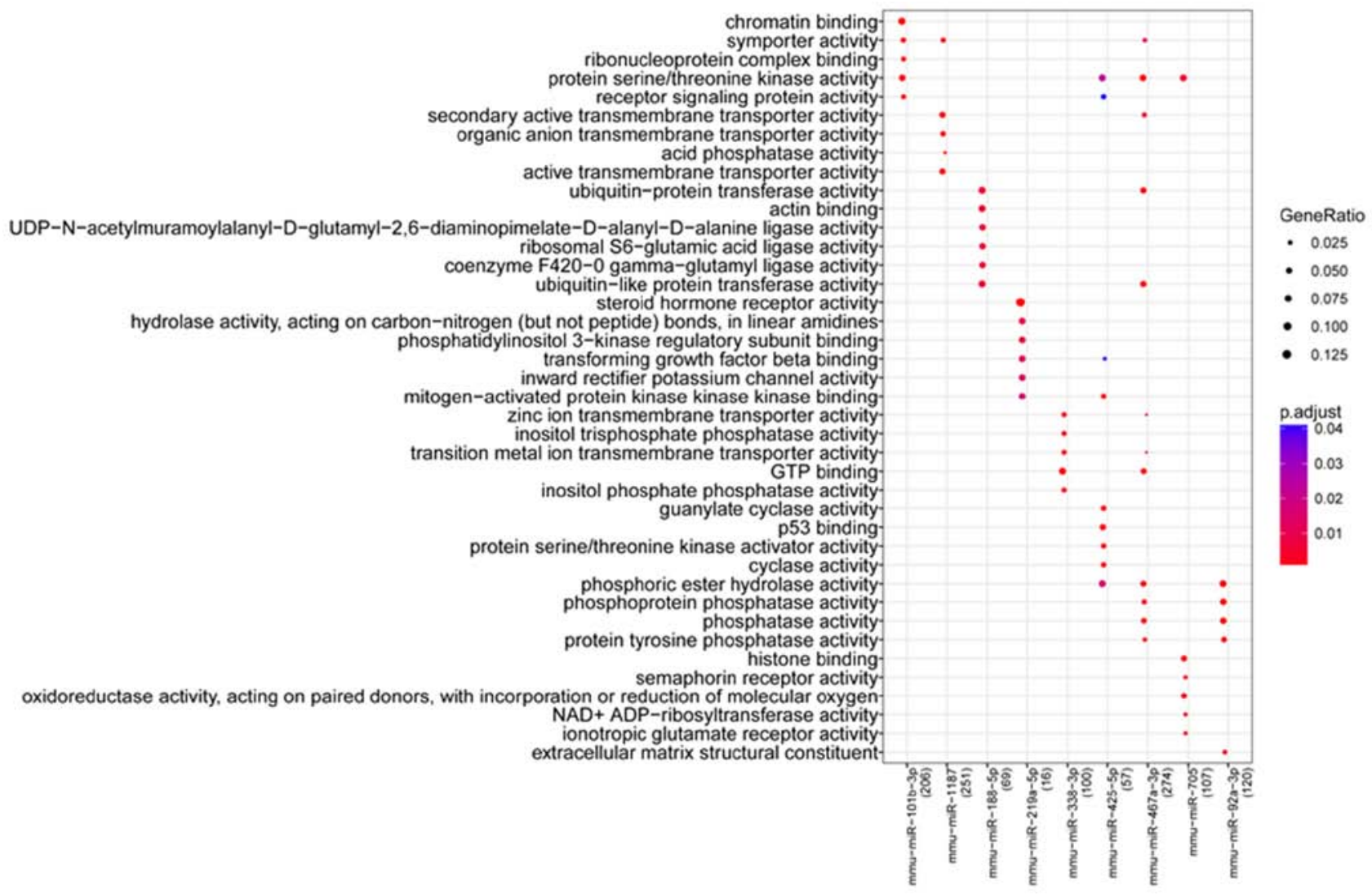

Figure 3. Significant Gene Ontology biological processes closely related with differentially expressed miRs. miR, microRNA.

Differentially expressed miRNAs. With $\mathrm{P}<0.01$ and $\log _{2} \mathrm{FCl}>0.263(18,19)$, the expression of 18 miRNAs, including 11 upregulated miRNAs (miR-1897-5p, miR-188-5p, miR-3098-5p, miR-3095-3p, miR-5107-5p, miR-3470a,
miR-705, miR-5126, miR-149-3p, miR-1187 and miR-1982-5p) and seven downregulated miRNAs (miR-425-5p, miR-101b-3p, miR-92a-3p, miR-338-3p, miR-467a-3p, miR-219-5p and miR-219-2-3p) were found to be significantly affected by 


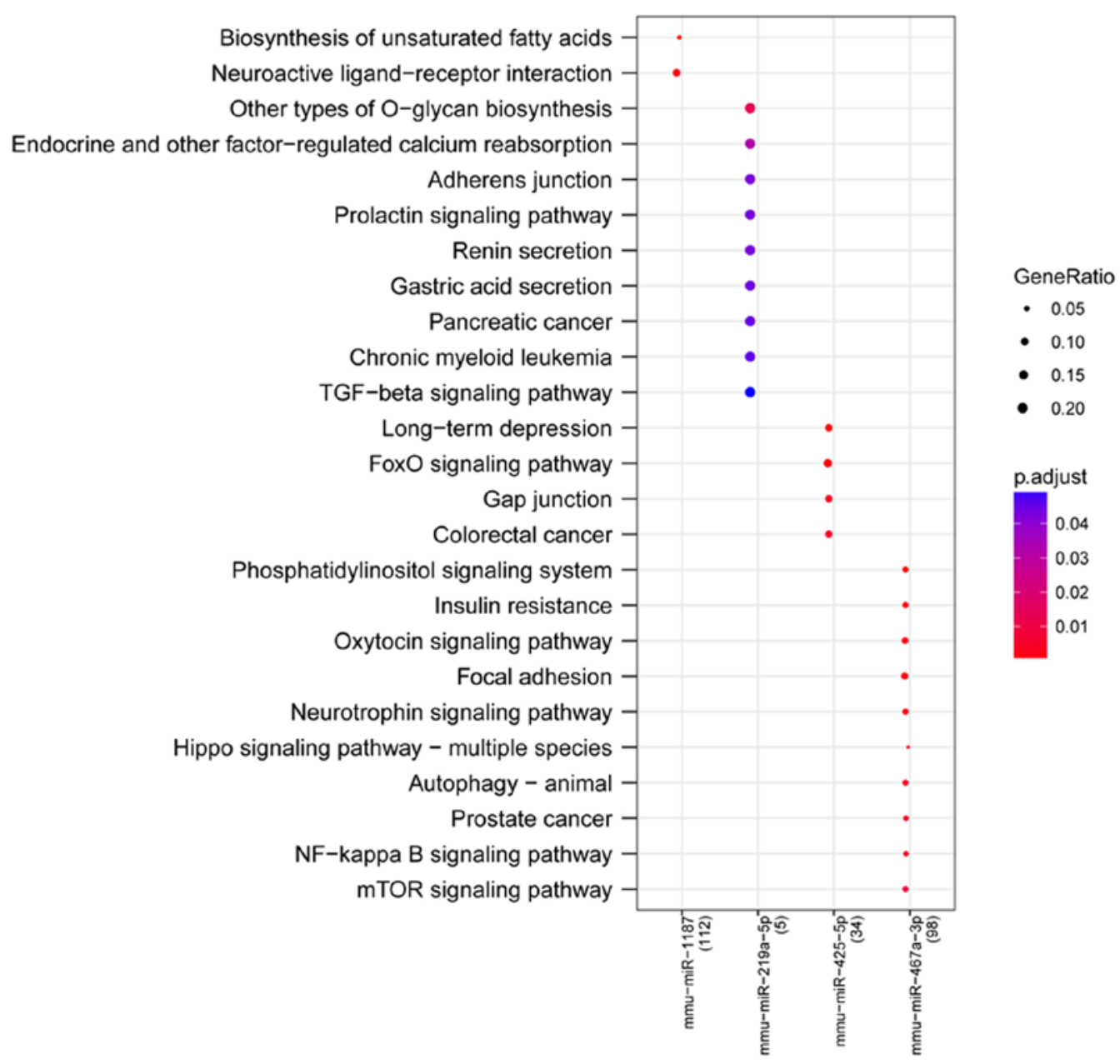

Figure 4. Pathways associated with differentially expressed miRs. miR, microRNA.

sevoflurane administration. The heatmap of differentially expressed miRNAs, which illustrate that the samples in different groups can be distinguished by the expression profiles of differentially expressed miRNAs, is presented in Fig. 1. Upregulated miRNAs in the sevoflurane group are shown in red, whereas downregulated miRNAs are presented as green.

miRNA-target genes and function enrichment analysis. Among 18 differentially expressed miRNAs, 3 upregulated miRNAs (miR-1187, miR-188-5p and miR-705) and 6 downregulated miRNAs (miR-101b-3p, miR-219a-5p, miR-338-3p, miR-425-5p, miR-467a-3p and miR-92a-3p) were identified to have 1,252 target genes based on the information of seven miRNA related databases. After the overlaps were removed, 1,095 target genes were obtained. Pathway enrichment analysis showed that the target genes were closely involved in the 'FoxO signaling pathway', 'mTOR signaling pathway' and 'Wnt signaling pathway' (Fig. 2A). Target genes clustered into different function groups such as 'dephosphorylation', 'vesicle localization' and the 'Wnt signaling pathway' (Fig. 2B).

miRNA-related functions and pathways. Based on the miRNA-target gene information, the biological function and pathways of the nine miRNAs were assessed by GO and pathway enrichment analysis. Results identified 190 biological process terms and 27 pathways significantly enriched by the nine miRNAs. Results showed that the biological functions and pathways closely related with miRNAs were relatively different. The top five GO function terms (ranked by P-value) of each miRNA are listed in Fig. 3. miR-101b-3p was closely related with 'chromatin binding' and 'protein serine/threonine kinase activity'. miR-1187 was significantly enriched in 'secondary active transmembrane transporter activity', 'organic anion transmembrane transporter activity' and 'active transmembrane transporter activity'. miR-219a-5p was involved in 'steroid hormone receptor activity', 'phosphatidylinositol 3-kinase regulatory subunit binding' and 'transforming growth factor- $\beta$ binding'. miR-467a-3p and miR-92a-3p were closely related with phosphatase activity.

Pathways that were particularly affected by four miRNAs (miR-1187, miR-219a-5p, miR-425-5p and miR-467a-3p) were screened out by KEGG signaling pathway enrichment analysis. The most significantly affected pathways for miRNAs included 'biosynthesis of unsaturated fatty acids' (miR-1187), 'neuroactive ligand-receptor interaction' (miR-1187), 'other types of O-glycan biosynthesis' (miR-219a-5p), 'long-term depression' (miR-425-5p), 'FoxO signaling pathway' (miR-425-5p), 'phosphatidylinositol signaling system' (miR-467a-3p), 'neurotrophin signaling pathway' (miR-467a-3p), 'Hippo signaling pathway-multiple species' and 'NF- $\kappa \mathrm{B}$ signaling pathway' (miR-467a-3p; Fig. 4). 


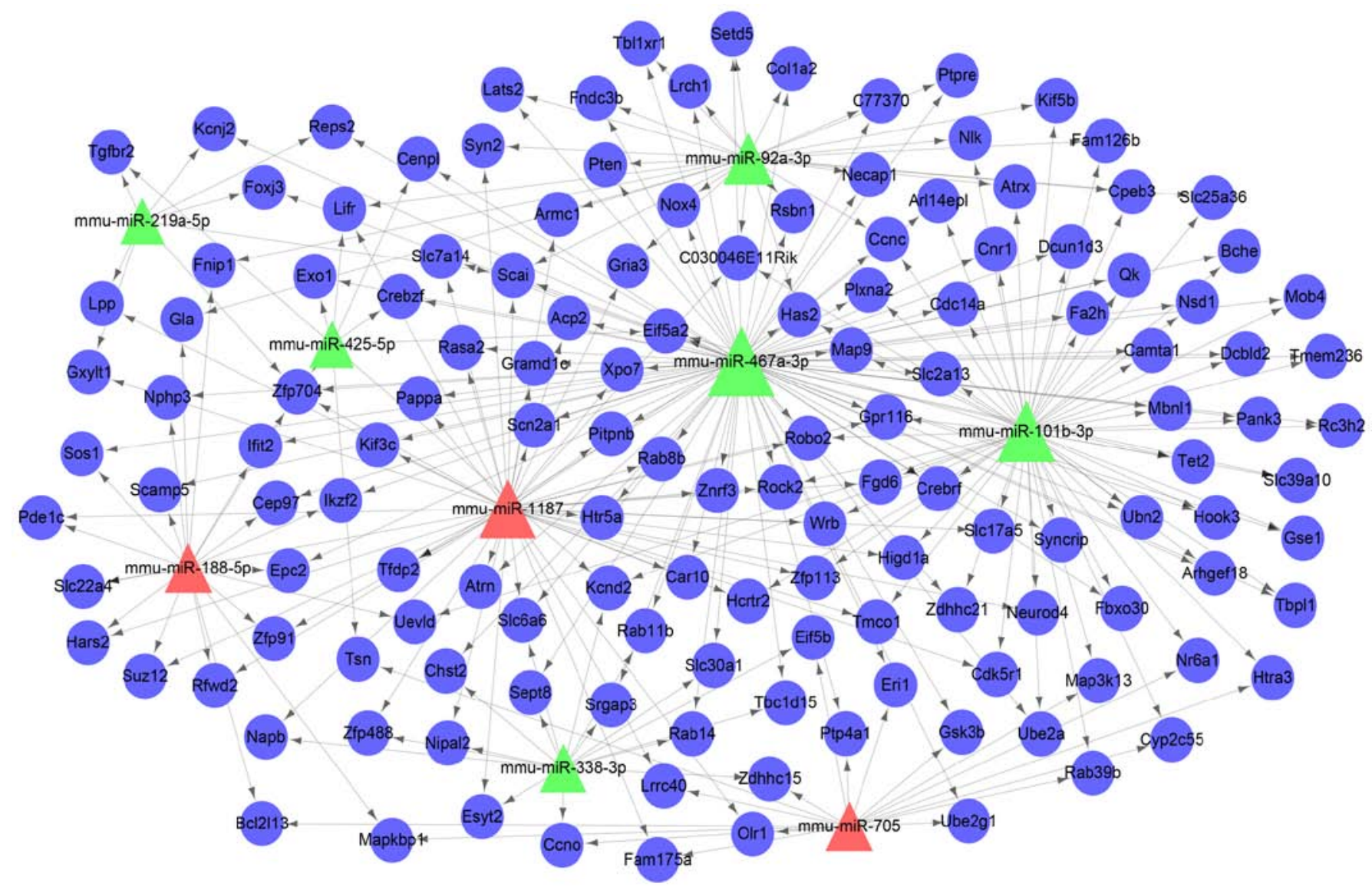

Figure 5. miRNA-target gene regulatory network. Green triangle, downregulated miRNA; red triangle, upregulated miRNA; blue circle, miRNA target genes. The size of the triangle nodes indicates the degree to which miRNAs contribute to the network. miRNA/miR, microRNA.

Table II. Top 10 nodes in the miR regulatory network.

\begin{tabular}{lcl}
\hline Name & Degree & Regulated \\
\hline miR-467a-3p & 89 & Down \\
miR-101b-3p & 59 & Down \\
miR-1187 & 51 & Up \\
miR-92a-3p & 28 & Down \\
miR-188-5p & 19 & Up \\
miR-705 & 18 & Up \\
miR-338-3p & 16 & Down \\
miR-425-5p & 9 & Down \\
miR-219a-5p & 8 & Down \\
Zfp704 & 5 & $/$ \\
\hline
\end{tabular}

miR, microRNA.

miRNA-target gene regulatory network. A miRNA regulatory network containing the nine miRNAs and 141 genes was constructed (Fig. 5). The top 10 nodes with the highest degrees were listed in Table II. The hub nodes included miR-467a-3p $($ degree $=89)$, miR-101b-3p $($ degree $=59)$, miR-1187 $($ degree $=51)$ and Zfp704 (degree=5).

\section{Discussion}

The alterations of the miRNA expression profile in the hippocampus tissues of mice exposed to sevoflurane may provide novel insight to understand the molecular mechanisms of memory and learning impairments resulting from altered development of the brain and allow opportunities to develop new therapeutic management strategies. The aim of this paper was to identify differentially expressed miRNAs in hippocampus tissues between mice exposed to sevoflurane and controls, and elucidate the molecular mechanisms that govern memory impairment in sevoflurane-exposed mice. The results identified 18 differentially expressed miRNAs. After function and pathway enrichment analysis, the target genes of differentially expressed miRNAs were found to be significantly enriched in the 'Wnt signaling pathway'.

A previous study also showed that the dysregulated miRNAs were closely related with Wnt signaling pathways underlying sevoflurane-induced neurotoxicity in the development of mice brains (31), which was consistent with the present results. Ye et al (31) predicted the gene targets for miRNAs with TargetScan, miRanda and PicTar. In the present study, the target genes for dysregulated miRNAs were predicted by miRWalk 2.0 combined with miRanda, miRDB, PITA, RNA22, RNAhybrid and TargetScan databases, which enhanced the accuracy of significant pathway identification 
and indicated the importance of the Wnt signaling pathway in the impaired cognition resulting from exposure to sevoflurane.

The Wnt signaling pathway is involved in various biological processes, such as cell proliferation, tissue regeneration, stem cell renewal and axon guidance $(15,32,33)$. It is reported that the Wnt signaling pathway is involved with $\sim 3 \%$ of differentially expressed transcripts that are prominent in the brain and spinal cord after spinal cord injury (SCI) in lampreys (34). Previous work has suggested an essential role for Wnt signaling in developing and adult brains (35). Blocking Wnt signaling has been shown to inhibit functional recovery following SCI (34), which indicates the significant role of Wnt signaling in neural repair and regeneration of the peripheral nervous system. In addition, synapse degeneration is closely associated with cognitive impairment and deficits in learning and memory. Evidence suggests that synapse degeneration, as an early event in neurodegenerative disease, is linked with Wnt signaling deficiency (36). The Wnt signaling pathway has been proposed as a therapeutic target for neuronal circuit recovery following synapse degeneration. A previous study also showed that reactivation of the Wnt signaling pathway improves neuroblast formation and neural function in the brain after focal cerebral ischemia in mice (37). Additionally, the Wnt signaling cascade is involved in the neuronal differentiation of human non-neural tissue-derived stem cells (35). Wnt signaling pathways are implicated in the differentiation of neural stem cells in human brain development (38). Taken together, these findings indicated a significant role of the Wnt signaling pathway in mediating cognitive disorders in brains exposed to sevoflurane.

The miRNA target gene regulatory network illustrated that miR-467a-3p (degree=89), miR-101b-3p (degree=59), and miR-1187 (degree=51) were hub nodes with multiple connections with target genes, which suggested a regulatory role for these miRNAs. The upregulated miR-188-5p (degree=19) was found to be another significant node in the miRNA regulatory network. A recent study suggested that miR-188-3p was upregulated in sevoflurane-treated mice and involved in sevoflurane-induced cognitive dysfunction (39), which was consistent with the present results. miR-188-5p is an alternative mature body of miR-188 and, to our knowledge, has not yet been reported to be dysregulated following sevoflurane exposure. It has been reported that miRNA-188-3p targeting mouse double minute 2 plays a significant role in sevoflurane-induced apoptosis pathways (39). The key role of miR-188-5p in miRNA-target gene regulatory networks may provide new insight into further gene targets in cognitive impairment induced by sevoflurane.

A recent study showed that overexpression of miR-467a-3p inhibits the neural differentiation of mouse embryonic stem cells (ESCs) (40). Neural stem cells differentiated from ESCs are suggested to be involved in cognition impairment-related diseases in humans $(41,42)$. miR-467a-3p has also previously been found to be involved in the apoptosis of vascular smooth muscle cells (43). The present study showed that miR-467a-3p was differentially expressed in the hippocampus tissues of mice exposed to sevoflurane, which indicated that the neural differentiation and proliferation were dysregulated upon sevoflurane exposure. In addition, the pathway enrichment analysis conducted in this study showed that miR-467a-3p was closely related with the neurotrophin signaling pathway. Neurotrophic factors are implicated in the development and maintenance of the nervous system (44). The dysregulation of neurotrophin signaling has been found to be associated with neurodegeneration in Alzheimer's disease (44). Taken together, these data suggested that miR-467a-3p may play a significant regulatory role in the maintenance of neuron function.

Furthermore, miR-1187 has been found to be a novel miRNA in the inhibition of osteoblast differentiation (45). miR-101b-3p has been found to be enriched in hepatocytes and is markedly upregulated following hepatocyte damage (46). Although evidence of the role of miR-1187 and miR-101b-3p in the regulation of plasticity in the hippocampus is lacking, the present study showed that miR-1187 was closely associated with 'neuroactive ligand-receptor interaction' and miR-101b-3p was closely related with 'receptor signaling protein activity'. The differential expression of miR-1187 and miR-101b-3p may impact neuroactive signaling interactions in brains exposed to sevoflurane.

In conclusion, the present findings suggested that the Wnt signaling pathway is involved in mediating cognitive disorder upon exposure to sevoflurane. miR-467a-3p may play a significant regulatory role in the maintenance of neuron function. miR-1187 and miR-101b-3p may be implicated in the regulation of neuroactive signaling interactions. The miRNAs and their related pathways may be important therapeutic targets to prevent sevoflurane-induced memory and learning disorders.

\section{Acknowledgements}

Not applicable.

\section{Funding}

This study was supported by Zhejiang Medical and Health Science and Technology Plan (grant no. 2014KYA159),Zhejiang Traditional Chinese Medicine Science and Technology Plan (grant no. 2019ZA047) and Natural Science Foundation of Zhejiang Province (grant no. LY20H150002).

\section{Availability of data and materials}

The datasets used and/or analyzed during the present study are available from the corresponding author on reasonable request.

\section{Authors' contributions}

HS and ZL conceived and designed the study; HH and XX acquired the data and analyzed and interpreted the data; TT performed the statistical analysis; HS drafted the manuscript; and $\mathrm{ZL}$ revised the manuscript for important intellectual content. All authors read and approved the final manuscript.

\section{Ethics approval and consent to participate}

Approval was obtained from Animal Care and Ethics Committee of Zhejiang Chinese Medical University and all the animal procedures were performed according to the institution's ethical standards. 


\section{Patient consent for publication}

Not applicable.

\section{Competing interests}

The authors declare that they have no competing interests.

\section{References}

1. Wang SQ, Fang F, Xue ZG, Cang J and Zhang XG: Neonatal sevoflurane anesthesia induces long-term memory impairment and decreases hippocampal PSD-95 expression without neuronal loss. Eur Rev Med Pharmacol Sci 17: 941-950, 2013.

2. Xiao H, Liu B, Chen Y and Zhang J: Learning, memory and synaptic plasticity in hippocampus in rats exposed to sevoflurane. Int J Dev Neurosci 48: 38-49, 2016.

3. Kamal A, Ramakers G, Gispen WH and Biessels GJ: Hyperinsulinemia in rats causes impairment of spatial memory and learning with defects in hippocampal synaptic plasticity by involvement of postsynaptic mechanisms. Exp Brain Res 226: 45-51, 2013

4. Liu XS, Xue QS, Zeng QW, Li Q, Liu J, Feng XM and Yu BW: Sevoflurane impairs memory consolidation in rats, possibly through inhibiting phosphorylation of glycogen synthase kinase-3 $\beta$ in the hippocampus. Neurobiol Learn Mem 94: 461-467, 2010.

5. Liu X, Song X, Yuan T, He J, Wang X and Wang Q: Effects of calpain on sevoflurane-induced aged rats hippocampal neuronal apoptosis. Aging Clin Exp Res 28: 633-639, 2016.

6. Ge X, Zhang Y, Zuo Y, Israr M, Li B, Yu P, Gao G, Chang YZ and Shi Z: Transcriptomic analysis reveals the molecular mechanism of Alzheimer-related neuropathology induced by sevoflurane in mice. J Cell Biochem 120: 17555-17565, 2019.

7. Liu Y, Gao M, Ma L, Zhang L and Pan N: Sevoflurane alters the expression of receptors and enzymes involved in $\mathrm{A} \beta$ clearance in rats. Acta Anaesthesiol Scand 57: 903-910, 2013.

8. Li Y, Liu L, Tian Y and Zhang J: Rapamycin improves sevoflurane-induced cognitive dysfunction in aged rats by mediating autophagy through the TLR4/MyD88/NF- $\mathrm{B}$ signaling pathway. Mol Med Rep 20: 3085-3094, 2019.

9. Xu B, Hsu PK, Karayiorgou M and Gogos JA: MicroRNA dysregulation in neuropsychiatric disorders and cognitive dysfunction. Neurobiol Dis 46: 291-301, 2012.

10. Maffioletti E, Tardito D, Gennarelli M and Bocchio-Chiavetto L: Micro spies from the brain to the periphery: New clues from studies on microRNAs in neuropsychiatric disorders. Front Cell Neurosci 8: 75, 2014.

11. Veltri A, Lang C and Lien WH: Concise review: Wnt Signaling pathways in skin development and epidermal stem cells. Stem Cells 36: 22-35, 2018.

12. Amjadi-Moheb F and Akhavan-Niaki H: Wnt signaling pathway in osteoporosis: Epigenetic regulation, interaction with other signaling pathways, and therapeutic promises. J Cell Physiol 234: 14641-14650, 2019.

13. Clevers $\mathrm{H}$ and Nusse $\mathrm{R}$ : Wnt/ $\beta$-catenin signaling and disease Cell 149: 1192-1205, 2012.

14. Mahmood S, Bhatti A, Syed NA and John P: The microRNA regulatory network: A far-reaching approach to the regulate the Wnt signaling pathway in number of diseases. J Recept Signal Transduct Res 36: 310-318, 2016.

15. Shih J, May LD, Gonzalez HE, Lee EW, Alvi RS, Sall JW, Rau V, Bickler PE, Lalchandani GR, Yusupova M, et al: Delayed environmental enrichment reverses sevoflurane-induced memory impairment in rats. Anesthesiology 116: 586-602, 2012.

16. Ishikawa M, Tanaka S, Arai M, Genda $Y$ and Sakamoto A: Differences in microRNA changes of healthy rat liver between sevoflurane and propofol anesthesia. Anesthesiology 117: $1245-1252,2012$

17. Su D, Zhao Y, Wang B, Xu H, Li W, Chen J and Wang X: Isoflurane-induced spatial memory impairment in mice is prevented by the acetylcholinesterase inhibitor donepezil. PLoS One 6: e27632, 2011.

18. Gautier L, Cope L, Bolstad BM and Irizarry RA: Affy-analysis of affymetrix GeneChip data at the probe level. Bioinformatics 20 307-315, 2004.

19. Bolstad BM, Irizarry RA, Astrand M and Speed TP: A comparison of normalization methods for high density oligonucleotide array data based on variance and bias. Bioinformatics 19: 185-193, 2003
20. Irizarry RA, Hobbs B, Collin F, Beazer-Barclay YD, Antonellis KJ, Scherf U and Speed TP: Exploration, normalization, and summaries of high density oligonucleotide array probe level data. Biostatistics 4: 249-264, 2003.

21. Ritchie ME, Phipson B, Wu DI, Hu Y, Law CW, Shi W and Smyth GK: Limma powers differential expression analyses for RNA-sequencing and microarray studies. Nucleic Acids Res 43: e47, 2015

22. Yu J,Zhu M, Lv M, Wu X, Zhang X, Zhang Y, Li J and Zhang Q: Characterization of a five-microRNA signature as a prognostic biomarker for esophageal squamous cell carcinoma. Sci Rep 9: $19847,2019$.

23. Zhang S, Liu W, Liu X, Qi J and Deng C: Biomarkers identification for acute myocardial infarction detection via weighted gene co-expression network analysis. Medicine (Baltimore) 96: e8375, 2017.

24. Breuer K, Foroushani AK, Laird MR, Chen C, Sribnaia A, Lo R, Winsor GL, Hancock REW, Brinkman FSL and Lynn DJ: InnateDB: Systems biology of innate immunity and beyond-recent updates and continuing curation. Nucleic Acids Res 41 (Database issue): D1228-D1233, 2013.

25. Dweep H and Gretz N: miRWalk2.0: A comprehensive atlas of microRNA-target interactions. Nat Methods 12: 697-697, 2015.

26. Ashburner M, Ball CA, Blake JA, Botstein D, Butler H, Cherry JM, Davis AP, Dolinski K, Dwight SS, Eppig JT, et al: Gene ontology: Tool for the unification of biology. The gene ontology consortium. Nat Genet 25: 25-29, 2000.

27. Kanehisa M and Goto S: KEGG: Kyoto encyclopaedia of genes and genomes. Nucleic Acids Res 28: 27-30, 2000.

28. Yu G, Wang LG, Han Y and He QY: clusterProfiler: An $\mathrm{R}$ package for comparing biological themes among gene clusters. OMICS 16: 284-287, 2012.

29. Shannon P, Markiel A, Ozier O, Baliga NS, Wang JT, Ramage D, Amin N, Schwikowski B and Ideker T: Cytoscape: A software environment for integrated models of biomolecular interaction networks. Genome Res 13: 2498-2504, 2003.

30. Jaksik R, Polańska J, Herok R and Rzeszowska-Wolny J: Calculation of reliable transcript levels of annotated genes on the basis of multiple probe-sets in affymetrix microarrays. Acta Biochim Pol 56: 271-277, 2009.

31. Ye J, Zhang Z, Wang Y, Chen $\mathrm{C}, \mathrm{Xu} \mathrm{X}, \mathrm{Yu} \mathrm{H}$ and Peng M: Altered hippocampal microRNA expression profiles in neonatal rats caused by sevoflurane anesthesia: MicroRNA profiling and bioinformatics target analysis. Exp Ther Med 12: 1299-1310, 2016.

32. Kyritsis N, Kizil C, Zocher S, Kroehne V, Kaslin J, Freudenreich D, Iltzsche A and Brand M: Acute inflammation initiates the regenerative response in the adult zebrafish brain. Science 338: 1353-1356, 2012.

33. MacDonald BT, Tamai K and He X: Wnt//-catenin signaling: Components, mechanisms, and diseases. Dev Cell 17: 9-26, 2009.

34. Herman PE, Papatheodorou A, Bryant SA, Waterbury CKM, Herdy JR, Arcese AA, Buxbaum JD, Smith JJ, Morgan JR and Bloom O: Highly conserved molecular pathways, including Wnt signaling, promote functional recovery from spinal cord injury in lampreys. Sci Rep 8: 742, 2018.

35. L'Episcopo F, Tirolo C, Caniglia S, Testa N, Morale MC, Serapide MF, Pluchino S and Marchetti B: Targeting Wnt signaling at the neuroimmune interface for dopaminergic neuroprotection/repair in parkinson's disease. J Mol Cell Biol 6: 13-26, 2014.

36. Marzo A, Galli S, Lopes D, McLeod F, Podpolny M, Segovia-Roldan M, Ciani L, Purro S, Cacucci F, Gibb A and Salinas PC: Reversal of synapse degeneration by restoring Wnt signaling in the adult hippocampus. Curr Biol 26: 2551-2561, 2016

37. Qiu CW, Liu ZY, Hou K, Liu SY, Hu YX, Zhang L, Zhang FL, Lv KY, Kang Q, Hu WX, et al: Wip1 knockout inhibits neurogenesis by affecting the Wnt/ $\beta$-catenin signaling pathway in focal cerebral ischemia in mice. Exp Neurol 309: 44-53, 2018.

38. Vangipuram SD and Lyman WD: Ethanol affects differentiation-related pathways and suppresses Wnt signaling protein expression in human neural stem cells. Alcohol Clin Exp Res 36: 788-797, 2012

39. Wang L, Zheng M, Wu S and Niu Z: MicroRNA-188-3p is involved in sevoflurane anesthesia-induced neuroapoptosis by targeting MDM2. Mol Med Rep 17: 4229-4236, 2018.

40. Zhang L, Xue Z, Yan J, Wang J, Liu Q and Jiang H: LncRNA Riken-201 and Riken-203 modulates neural development by regulating the Sox6 through sequestering miRNAs. Cell Prolif 52: e12573, 2019. 
41. De Filippis L,Zalfa C and Ferrari D: Neural stem cells and human induced pluripotent stem cells to model rare CNS diseases. CNS Neurol Disord Drug Targets 16: 915-926, 2017.

42. Sugaya K and Vaidya M: Stem cell therapies for neurodegenerative diseases. Adv Exp Med Biol 1056: 61-84, 2018.

43. Cui R, Ye S, Zhong J, Liu L, Li S, Lin X, Yuan L and Yi L: MicroRNA-494 inhibits apoptosis of murine vascular smooth muscle cells in vitro. Mol Med Rep 19: 4457-4467, 2019.

44. Chen XQ, Sawa M and Mobley WC: Dysregulation of neurotrophin signaling in the pathogenesis of alzheimer disease and of alzheimer disease in down syndrome. Free Radic Biol Med 114: 52-61, 2018.

45. John AA, Prakash R, Kureel J and Singh D: Identification of novel microRNA inhibiting actin cytoskeletal rearrangement thereby suppressing osteoblast differentiation. J Mol Med (Berl) 96: 427-444, 2018
46. Takeuchi M, Oda S, Tsuneyama K and Yokoi T: Comprehensive analysis of serum microRNAs in hepatic sinusoidal obstruction syndrome (SOS) in rats: Implication as early phase biomarkers for SOS. Arch Toxicol 92: 2947-2962, 2018.

(i) $($ ) This work is licensed under a Creative Common Attribution-NonCommercial-NoDerivatives 4.0 International (CC BY-NC-ND 4.0) License. 